\title{
The Viral Invasion - Unusual Presentation of Herpes Simplex Keratitis in a Patient with SARS-CoV-2 Infection
}

\section{Krunal Patel ${ }^{1}$ and Anuja K Desai ${ }^{2 *}$}

${ }^{1}$ Phaco Refractive Surgeon, Crystal Eye Care, Ahmedabad, Gujarat, India ${ }^{2}$ Cornea and Refractive Surgeon, Department of Ophthalmology, Kusum Dhirajlal Hospital, Ahmedabad, Gujarat, India

*Corresponding Author: Anuja K Desai, Cornea and Refractive Surgeon, Department of Ophthalmology, Kusum Dhirajlal Hospital, Ahmedabad, Gujarat, India.
Received: October 07, 2021

Published: October 15, 2021

(C) All rights are reserved by Krunal Patel and Anuja K Desai.

\begin{abstract}
Background: To report a case of herpes simplex viral (HSV-1) keratitis secondary to COVID-19 infection.

Case Presentation: A 55-year-old man developed ocular symptoms after recovering from SARS- CoV-2 infection. After initial assessment a diagnosis of HSV-1 keratitis was confirmed and treated with topical antiviral. The complete reduction of keratitis symptoms and a clear cornea was achieved within 4 weeks.

Conclusions: There have been multiple reports on varied ocular manifestations of COVID-19 infection. This case highlights the possibility of SARS-CoV-2 acting as a potential trigger for viral reactivation.
\end{abstract}

Keywords: COVID-19; HSV-1 Keratitis; SARS-CoV-2; Immunocompromised

\section{Abbreviations}

HSV-1: Herpes Simplex Viral Type 1; HSV-2: Herpes Simplex Virus Type 2; BCVA: Best-corrected Visual Acuity.

\section{Introduction}

Herpes simplex keratitis is a recurrent infection of the cornea caused by herpes simplex virus type 1 (HSV-1) and less frequently by herpes simplex virus type 2 (HSV-2) [1]. The incidence of Herpes simplex keratitis in developed countries is estimated to be between 10 and 30 per 100,000 of the population per year with a prevalence of 149 per 100,000 . The incidence and prevalence are thought to be higher in developing countries [2]. Relapses are common with $40 \%$ of patients experiencing $2-5$ relapses in a lifetime and 11\% experiencing 6-15 relapses [3]. This virus has a propensity to affect immunosuppressed individuals, patients with psychological stress and hormonal changes [4].

Coronaviruses can cause severe ocular disease in animals, including anterior uveitis, retinitis, vasculitis, and optic neuritis in feline and murine species. However, ocular manifestations in humans are typically mild and rare, although there are increasing numbers of associated ocular findings in patients positive for the COVID-19 [5].

\section{Case Description}

A 55-year-old male patient presented with a three-day history of watering, redness, burning sensation and occasional pain in the 
left eye. The patient had tested positive for SARS-CoV-2 using the reverse transcription (RT) polymerase chain reaction (PCR) test 15 days prior. During his course of infection, he was hospitalized for ten days; intravenous injection Remdesivir was given for five days, supplemental oxygen administered for one day. No history of steroid administration.

Upon ocular examination, both eyes best-corrected visual acuity (BCVA) was 20/20. The left lower eyelid had a small painful swelling with black crusting over the lesion and surrounding purulent discharge. Eye movements were free, full and painless in all directions. The anterior segment was within normal limits except mild conjunctival hyperemia nasally. The right eye was within normal limits. Both eyes posterior segments were normal. Crusting over the lower lid was removed using a sterile bud and local application of moxifloxacin (0.5\%) eye ointment four times per day, and carboxymethylcellulose eye drop six times per day was prescribed. The patient was referred to an Otorhinolaryngologist to rule out any signs of mucormycosis. Nasal Endoscopy and MRI brain with orbit were normal.

Two days later, the patient developed severe pain and redness in the left eye. There was a definite reduction in the size of eyelid swelling with the absence of crusting and discharge. Slit-lamp examination revealed typical HSV-1 dendritic lesions throughout the cornea with the presence of fluorescein staining (Figure 1). Patient was started on acyclovir (3\%) eye ointment five times a day and carboxymethylcellulose eye drops six times/day. No past history of ocular/systemic herpes infection or chickenpox. The dendrites resolved within the next 48 hours, and the patient was symptomatically better. During this course, he developed a fever and was referred to a physician. His $C$ reactive protein was 25 (mg/L), and he was started on Intravenous meropenem. The patient stopped using acyclovir eye ointment on his own and came back a day later with a recurrence of multiple dendrites over the cornea.

The patient was counselled about the regular use of medications. One week later, the eye was quiet, keratitis resolved, diffuse dry spots all over the cornea (Figure 2), and vision restored. Four weeks after the initial presentation, the patient was symptomatically better; BCVA was 20/20 with a clear cornea. He was asked to continue lubricants and follow-up regularly.

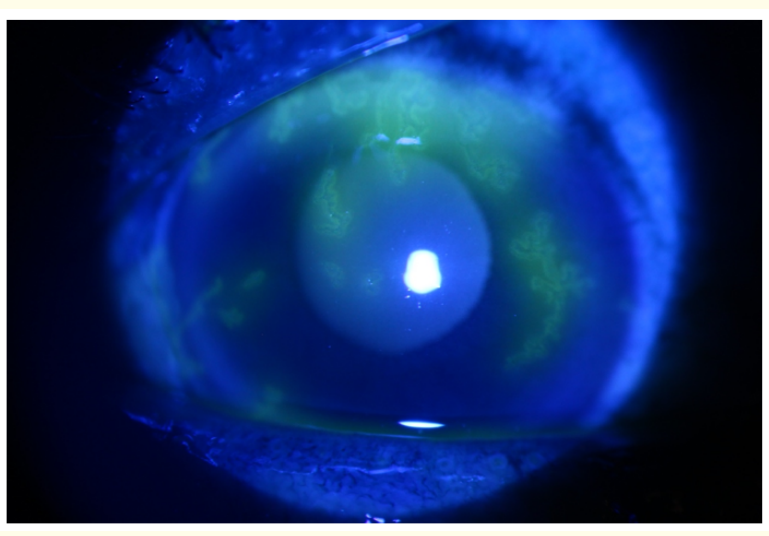

Figure 1: Peripheral HSV dendrites.

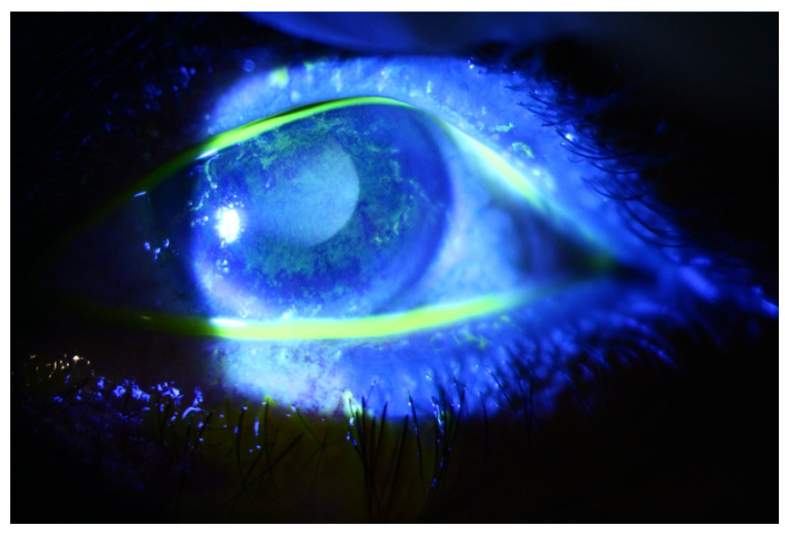

Figure 2: Multiple diffuse superficial punctate keratitis.

\section{Discussion and Conclusions}

COVID-19 was declared a public health emergency of international concern by WHO on $30^{\text {th }}$ January 2020, and a pandemic on $11^{\text {th }}$ March 2020 [6]. Since then, multiple pieces of research have taken place concerning the disease and its effect on the human body. The prevalence of ophthalmic manifestations among COVID-19 patients ranges from 2-32\% [7]. A 2021 metaanalysis by Nasiri., et al. [8] reported a pooled prevalence of all 
ocular manifestations among 7,300 COVID-19 patients as $11.03 \%$, with the most frequent ocular disease being conjunctivitis $(88.8 \%)$ followed by dry eye or foreign body sensation (16\%), eye redness $(13.3 \%)$, tearing $(12.8 \%)$, and itching $(12.6 \%)$ were among the most frequent symptoms reported.

In our case, the typical herpetic epithelial dendrites all over the cornea helped in timely diagnosis and treatment rather than primarily treating it as viral conjunctivitis. Reactivation of HSV secondary to immunosuppression was our initial thought, as SARSCoV-2 is known to cause a failure of antiviral immunity in COVID-19 infected individuals by decreasing the number and efficacy of T-cell lymphocytes and natural killer cells [9]. Majtanova., et al. [10] reported clinically confirmed HSV keratitis in 5 patients, with a majority of these patients having associated systemic comorbidities. Hernandez., et al. [11] also reported a case of HSV keratitis in an elderly patient with multiple systemic risk factors leading to HSV reactivation. However, our patient did not have any comorbidities or risk factors, except tobacco chewing vice. Our patient was not administered any of the systemic medications used in treating COVID 19 infection, which is known to have ocular side effects [7]. The lack of such common reasons for HSV reactivation in our case highlights the fact that SARS-CoV-2 infection induced immunosuppression could be the only cause of epithelial keratitis rather than an immune-mediated stromal inflammation as seen in cases of recurrence [4].

The ophthalmic features can develop at any point in the disease course. The median time of appearance from the development of COVID-19 symptoms/diagnosis of neuro-ophthalmic features is five days, of the ocular surface and anterior segment manifestations are 8.5 days, and posterior segment and orbital pathology are 12 days [7]. There is a possibility of COVID-19 infection in various organs, including the eye; hence thorough evaluation of even a minor symptom in the setting of COVID-19 disease is of paramount importance.

As COVID-19 is known to humanity for the last 1.5 years only, there are still many unanswered questions. SARS-CoV-2 infection may be a risk factor for developing HSV-1 keratitis or may act as a potential activator of this ocular disease. With the recent mutation of the virus, there is an imminent need for population-based studies to standardize identification and treatment protocols.

\section{Conflict of Interest}

Nil.

\section{Disclosure of any Funding Received for this Work}

Nil.

\section{Authors Contribution}

- KP- Diagnosed and examined the patient.

- $\quad$ AD- Guided for further course of treatment.

- All authors read and approved the final manuscript.

Availability of Data and Materials

Not applicable.

\section{Bibliography}

1. Looker KJ., et al. "Global and regional estimates of prevalent and incident herpes simplex virus type 1 infections in 2012". PloS one 10.10 (2015): e0140765.

2. Sibley D and Larkin DF. "Update on Herpes simplex keratitis management". Eye 34.12 (2020): 2219-2226.

3. Wishart MS., et al. "Recurrent herpes simplex virus ocular infection: epidemiological and clinical features". British Journal of Ophthalmology 71.9 (1987): 669-672.

4. Rolinski J and Hus I. "Immunological aspects of acute and recurrent herpes simplex keratitis". Journal of Immunology Research (2014).

5. Hu K., et al. "Ophthalmic manifestations of coronavirus (COVID-19)". StatPearls (2021).

6. who.int/news/item/27-04-2020-who-timeline---covid-19

7. Sen M., et al. "COVID-19 and eye: A review of ophthalmic manifestations of COVID-19". Indian Journal of Ophthalmology 69.3 (2021): 488.

8. Nasiri N., et al. "Ocular manifestations of COVID-19: A systematic review and meta-analysis". Journal of Ophthalmic and Vision Research 16.1 (2021): 103.

9. Heidary F., et al. "Acyclovir as a potential add-on treatment for COVID-19: A narrative review" (2021). 
10. Majtanova N., et al. "Herpes Simplex Keratitis in Patients with SARS-CoV-2 Infection: A Series of Five Cases". Medicina 57.5 (2021): 412 .

11. Hernandez JM., et al. "SARS-CoV-2 Infection (COVID-19) and Herpes Simplex Virus-1 Conjunctivitis: Concurrent Viral Infections or a Cause-Effect Result?". Cureus 13.1 (2021).

Volume 4 Issue 11 November 2021

(C) All rights are reserved by Krunal Patel and Anuja K Desai. 